International Journal of Pure and Applied Mathematics

Volume 87 No. 4 2013, 669-680

ISSN: 1311-8080 (printed version); ISSN: 1314-3395 (on-line version)

url: http://www.ijpam.eu

doi: http://dx.doi.org/10.12732/ijpam.v87i4.15

ijpam.eu

\title{
BLOCK EMPIRICAL LIKELIHOOD FOR PARTIALLY \\ INEAR ERRORS-IN-VARIABLES MODELS \\ WITH LONGITUDINAL DATA
}

\author{
Yafeng $\mathrm{Xia}^{1}, \mathrm{Hu} \mathrm{Da}^{2} \S$ \\ ${ }^{1,2}$ School of Science \\ Lanzhou University of Technology \\ Lanzhou, Gansu, 730050, P.R. CHINA
}

\begin{abstract}
In this paper, block empirical likelihood inference for partially linear errors-in-variables models with longitudinal data are investigated. We apply the block empirical likelihood procedure to accommodate the withingroup correlation of the longitudinal data. The block empirical log-likelihood ratio statistic for the parametric components, which are of primary interest, is suggested. And the nonparametric version of the Wilk's theorem is derived under mild conditions. Thus, the empirical likelihood confidence region with asymptotically correct coverage probabilities for parametric components can be constructed. Simulations are carried out to access the performance of the proposed approach.
\end{abstract}

AMS Subject Classification: $62 \mathrm{G} 15,62 \mathrm{G} 20$

Key Words: block empirical likelihood, partially linear model, errors-invariables, longitudinal data

\section{Introduction}

Longitudinal data arise frequently in epidemiological and economical studies,

Received: August 26, 2013

(c) 2013 Academic Publications, Ltd.

$\S$ Correspondence author url: www.acadpubl.eu 
and various methods for analysing these data have been proposed. For longitudinal data, an useful semiparametric models is partially linear model which has the following form:

$$
Y(t)=X(t)^{\tau} \beta+\theta(t)+\epsilon(t)
$$

where $Y(t)$ is the response variable, $X$ and $t$ are regressors, $\theta(t)$ is an arbitrary smooth functions of time $t, \beta=\left(\beta_{1}, \ldots, \beta_{p}\right)^{\tau}$ is a $p$-dimensional vector of unknown parameters, $\epsilon(t)$ is a zero-mean stochastic process. Due to the curse of dimensionality, for simplicity, we assume that $t$ is univariate. Here, $t$ ranges over a nondegenerate compact interval, without loss of generality, assumed to be the unit interval $[0,1]$.

Obviously, model (1) has been studied by many authors. Engle et al. (1986) [1] used to analyze the relation between weather and electricity sales. $\mathrm{Hu}$ et al. (2004) [2] studied the profile kernel and backfitting methods and further studied by Heckman (1986) [3], Chen and Shiau (1991) [4].

However, in many practical situations, these variables are often measured with error. In this paper, for model (1) we consider in this case where the variable $X(t)$ is measured with additive error. That is, $X(t)$ can not be observed, but an unbiased measure of $X(t)$, denoted by $W(t)$, can be obtained as follows

$$
W(t)=X(t)+U(t)
$$

where $U(t)$ is the measurement error, which is independent of $\left(X^{\tau}(t), \epsilon(t)\right.$, $t$ ), with mean zero and covariance matrix $\Sigma_{u u}$. We can assume that $\Sigma_{u u}$ is known. If $\Sigma_{u u}$ is unknown, we also can estimate it by repeatedly measuring $W(t)$ by Liang et al. (1999) [5]. For errors-in-variables models, Liang et al. (2000) [6] and Zhu and Cui (2003) [7] stuied the partially linear model when the nonparametric part is measured with error.

The empirical likelihood, which is a nonparametric approach for constructing confidence regions, was introduced by Owen (1988) [8] and has many nice statistical properties (see Owen (1990) [9]). Owen (1991) [10] applied empirical likelihood to linear regression models . Recently, Shi and Lau (2000) [11],Wang and Jing (2003) [12] considered the partial linear models. Xue and Zhu (2007) [13], Li and Xue (2008) [14] investigated the empirical likelihood confidence regions for a partially linear models with longitudinal data. Other related papers contain Cui and Chen (2003) [15], You et al. (2006) [16], Xue and Zhu (2008) [17] and $\mathrm{Lu}(2009)$ [18].

In this paper, we consider model (1)-(2) with longitudinal data, one aim of this paper is to construct the confidence region for the parameter components. 
To achieve it, we apply the block empirical likelihood approach [16] to construct block empirical log-likelihood ratio statistic for parameter $\beta$, and then prove the nonparametric Wilk's phenomenon. Simulation studies assess the proposed method.

The rest of this paper is organized as follows: In Section 2, we construct the block empirical likelihood based confidence region for the parametric components. Assumption conditions and main results are given in Section 3. Simulation results are reported in Section 4. The proofs of the main results are stated in Section 5.

\section{Methodology}

In this section, we are to extend the result of Li (2008) [14] to partially linear errors-in-variables model with longitudinal data. We apply longitudinal data $\left\{Y_{i}\left(t_{i j}\right), X_{i}\left(t_{i j}\right), t_{i j}\right\}$ which are generated from the following equation:

$$
\left\{\begin{aligned}
Y_{i j} & =X_{i j}^{\tau} \beta+\theta\left(t_{i j}\right)+\epsilon_{i j} \\
W_{i j} & =X_{i j}+U_{i j}
\end{aligned}\right.
$$

where $Y_{i j}=Y_{i}\left(t_{i j}\right), X_{i j}=X_{i}\left(t_{i j}\right), \epsilon_{i j}=\epsilon_{i}\left(t_{i j}\right)$ and $U_{i j}=U_{i}\left(t_{i j}\right), i=$ $1, \ldots, n, j=1, \ldots, n_{i}$. We assume in our asymptotic study that $n_{i}$ is bounded, but the number of subjects $n$ goes to infinity.

Suppose that $\beta$ is known, then model (3) can be reduced to a the nonparametric regression model.

$$
Y_{i j}-X_{i j}^{\tau} \beta=\theta\left(t_{i j}\right)+\epsilon_{i j}, \quad i=1, \ldots, n, j=1, \ldots, n_{i}
$$

Here, the local linear regression method is applied to estimate $\theta(\cdot)$ in model (4). That is, for $t$ in a small neighborhood of $t_{0}$, one can approximate $\theta(\cdot)$ locally by a linear function

$$
\theta(t) \approx \theta\left(t_{0}\right)+\theta^{\prime}\left(t_{0}\right)\left(t-t_{0}\right) \equiv a+b\left(t-t_{0}\right)
$$

where $\theta^{\prime}(t)=\partial \theta(t) / \partial t$. This leads to the following weighted least-squares problem: find $\{a, b\}$ to minimize.

$$
\sum_{i=1}^{n} \sum_{j=1}^{n_{i}}\left[Y_{i j}-X_{i j}^{\tau} \beta-\left\{a+b\left(t_{i j}-t_{0}\right)\right\}\right]^{2} K_{h}\left(t_{i j}-t\right)
$$

where $K$ is a kernel function, $K_{h}(\cdot)=K(\cdot / h) / h$ and $h$ is a bandwidth. Let 


$$
\begin{aligned}
& X=\left(\begin{array}{c}
X_{11}^{\tau} \\
\vdots \\
X_{1 n_{1}}^{\tau} \\
\vdots \\
X_{n n_{n}}^{\tau}
\end{array}\right)=\left(\begin{array}{ccc}
X_{111} & \cdots & X_{11 p} \\
\vdots & \ddots & \vdots \\
X_{1 n_{1} 1} & \cdots & X_{1 n_{1} p} \\
\vdots & \ddots & \vdots \\
X_{n n_{n} 1} & \cdots & X_{n n_{n} p}
\end{array}\right) \text {, } \\
& \Omega_{t}=\operatorname{diag}\left(K_{h}\left(t_{11}-t\right), \cdots, K_{h}\left(t_{1 n_{1}}-t\right), \cdots, K_{h}\left(t_{n n_{n}}-t\right)\right) \\
& Y=\left(Y_{11}, \cdots, Y_{1 n_{1}}, \cdots, Y_{n n_{n}}\right)^{\tau}, \epsilon=\left(\epsilon_{11}, \cdots, \epsilon_{1 n_{1}}, \cdots, \epsilon_{n n_{n}}\right)^{\tau} \\
& D_{t}=\left(\begin{array}{ccccc}
1 & \cdots & 1 & \cdots & 1 \\
h^{-1}\left(t_{11}-t\right) & \cdots & h^{-1}\left(t_{1 n_{1}}-t\right) & \cdots & h^{-1}\left(t_{n n_{n}}-t\right)
\end{array}\right)^{\tau}
\end{aligned}
$$

Then the solution to problem (6) is given by

$$
(\hat{a}(t), h \hat{b}(t))^{\tau}=\left(D_{t}^{\tau} \Omega_{t} D_{T}^{\tau}\right)^{-1} D_{t}^{\tau} \Omega_{t}\left(Y-X^{\tau} \beta\right)
$$

Then $\hat{\theta}(t)$ can be given by

$$
\hat{\theta}(t)=(1,0)\left(D_{t}^{\tau} \Omega_{t} D_{T}^{\tau}\right)^{-1} D_{t}^{\tau} \Omega_{t}\left(Y-X^{\tau} \beta\right)
$$

Denote

$$
(1,0)\left(D_{t}^{\tau} \Omega_{t} D_{T}^{\tau}\right)^{-1} D_{t}^{\tau} \Omega_{t} \equiv\left(S_{11}(t), \ldots, S_{n n_{n}}(t)\right),
$$

where

$$
S_{l m}(t)=\frac{N^{-1} K_{h}\left(t_{i j}-t\right)\left\{A_{k, 2}(t)-\left(t_{i j}-t\right) A_{k, 1}(t)\right\}}{A_{k, 0}(t) A_{k, 2}(t)-A_{k, 1}^{2}(t)}
$$

where $N=n_{1}+\cdots+n_{n}$,

$$
A_{k, s}=\frac{1}{N} \sum_{l=1}^{n} \sum_{m=1}^{n_{l}} K_{h}\left(t_{i j}-t\right)\left(t_{i j}-t\right)^{s}, \quad s=0,1,2
$$

then

$$
\hat{\theta}\left(t_{i j}\right)=\sum_{l=1}^{n} \sum_{m=1}^{n_{l}} S_{l m}\left(t_{i j}\right)\left(Y_{l m}-X_{l m}^{\tau} \beta\right)
$$

Substituting Equation (12) into Equation (4), we can obtain the approximate residuals as the following:

$$
\begin{aligned}
\hat{r}_{i j}(\beta) & =Y_{i j}-X_{i j}^{\tau} \beta-\sum_{l=1}^{n} \sum_{m=1}^{n_{l}} S_{l m}\left(t_{i j}\right)\left(Y_{l m}-X_{l m}^{\tau} \beta\right) \\
& =\tilde{Y}_{i j}-\tilde{X}_{i j}^{\tau} \beta
\end{aligned}
$$

where 


$$
\tilde{Y}_{i j}=Y_{i j}-\sum_{l=1}^{n} \sum_{m=1}^{n_{l}} S_{l m}\left(t_{i j}\right) Y_{l m}
$$

and

$$
\tilde{X}_{i j}=X_{i j}-\sum_{l=1}^{n} \sum_{m=1}^{n_{l}} S_{l m}\left(t_{i j}\right) X_{l m}
$$

Similar to Owen [10], $\left\{\hat{r}_{i j}(\beta), i=1, \ldots, n ; j=1, \ldots, n_{i}\right\}$ can be treated as a random sieve approximation of the random error sequence $\left\{\epsilon_{i j}, i=1, \ldots, n ; j=\right.$ $\left.1, \ldots, n_{i}\right\}$. In order to deal with the correlation within groups, we use the block empirical likelihood method. The block empirical likelihood procedure take the "data" $\hat{r}_{i j}(\beta), j=1, \ldots, n_{i}$ into account as a whole. Hence, to construct the block empirical likelihood ratio function for $\beta$, similar to Xue and Zhu [13], we introduce the auxiliary random vector

$$
\tilde{\eta}_{i}(\beta)=\sum_{j=1}^{n_{i}} \tilde{X}_{i j}\left[\tilde{Y}_{i j}-\tilde{X}_{i j}^{\tau} \beta\right]
$$

Following (14), if $\beta$ is true, then $E\left\{\tilde{\eta}_{i}(\beta)\right\}=o(1)$. If one ignores the measurement error and replaces $X_{i j}$ by $W_{i j}$ in $\tilde{\eta}_{i}(\beta)$, one can show that the resulting estimator is inconsistent. As we all know, inconsistency caused by the measurement error can be overcome by applying the so-called correction for attenuation proposed by Fuller [19] in linear regression. With a similar way as in You et al. [16], so the corrected-attenuation auxiliary vector is introduced and defined as

$$
\check{\eta}_{i}(\beta)=\sum_{j=1}^{n_{i}}\left\{\tilde{W}_{i j}\left(\tilde{Y}_{i j}-\tilde{W}_{i j}^{\tau} \beta\right)+\Sigma_{u u} \beta\right\}
$$

where $\tilde{W}_{i j}=W_{i j}-\sum_{l=1}^{n} \sum_{m=1}^{n_{l}} S_{l m}\left(t_{i j}\right) W_{l m}$. The term $\Sigma_{u u} \beta$ aims to avoid the underestimating for the parameter caused by the measurement error. Therefore, the empirical likelihood ratio function for $\beta$ defined as

$$
\mathscr{R}(\beta)=\max \left\{\prod_{i=1}^{n}\left(n p_{i}\right) \mid p_{i} \geq 0, \sum_{i=1}^{n} p_{i}=1, \sum_{i=1}^{n} p_{i} \check{\eta}_{i}(\beta)=0\right\}
$$

A unique value for $\mathscr{R}(\beta)$ exists, provided that 0 is inside the convex hull of the point $\left(\check{\eta}_{1}(\beta), \ldots, \check{\eta}_{n}(\beta)\right)$. Using the Lagrange multiplier technique, the optimal value for $p_{i}$ is

$$
p_{i}=\frac{1}{n}\left\{1+\lambda^{\tau} \check{\eta}_{i}(\beta)\right\}^{-1}, \quad i=1, \ldots, n
$$


where $\lambda=\left(\lambda_{1}, \ldots, \lambda_{n}\right)^{\tau}$ is the solution of the equation

$$
\frac{1}{n} \sum_{i=1}^{n} \frac{\check{\eta}_{i}(\beta)}{1+\lambda^{\tau} \check{\eta}_{i}(\beta)}=0
$$

Then, the block empirical log-likelihood ratio function is

$$
\mathscr{L} \mathscr{R}(\beta)=-2 \log \mathscr{R}(\beta)=2 \sum_{i=1}^{n} \log \left(1+\lambda^{\tau} \check{\eta}_{i}(\beta)\right)
$$

In addition, by maximizing $\mathscr{L} \mathscr{R}(\beta)$ we can obtain the maximum empirical likelihood estimator (MELE) $\check{\beta}$. Let

$$
\check{\Gamma}=\frac{1}{n} \sum_{i=1}^{n} \sum_{j=1}^{n_{i}}\left(\tilde{W}_{i j} \tilde{W}_{i j}^{\tau}-\Sigma_{u u}\right) .
$$

If the matrix $\check{\Gamma}$ is invertible, then the MELE of $\beta$ can be given by

$$
\check{\beta}=\check{\Gamma}^{-1} \frac{1}{n} \sum_{i=1}^{n} \sum_{j=1}^{n_{i}} \tilde{W}_{i j} \tilde{Y}_{i j}+o_{p}\left(n^{-1 / 2}\right)
$$

According to $\check{\beta}$, we can define the estimator $\theta(t)$ as

$$
\check{\theta}(t)=(1,0)\left(D_{t}^{\tau} \Omega_{t} D_{T}^{\tau}\right)^{-1} D_{t}^{\tau} \Omega_{t}\left(Y-X^{\tau} \check{\beta}\right)
$$

\section{Main Results}

To establish asymptotic properties of the block empirical log-likelihood ratio with making the following assumptions. These assumptions are quite mild and can be easily satisfied and founded in You et al. [16].

A1 The random variable $t$ has a compact support $\Xi$. The density function $f(\cdot)$ of $t$ has a continuous second derivative and is uniformly bounded away from zero.

A2 The $p \times p$ matrix $E\left(X X^{\tau} \mid t\right)$ is nonsingular and Lipschitz continuous for each $t \in \Xi$.

A3 There is a $s>2$ such that $E\|X\|^{2 s}<\infty, E\|\epsilon\|^{2 s}<\infty$ and $E\|t\|^{2 s}<\infty$, and for some $\epsilon<2-s^{-1}$ such that $n^{2 \epsilon-1} h \rightarrow \infty$ as $n \rightarrow \infty$, where $\|\cdot\|$ is Euclidean norm. 
A4 $\theta(\cdot)$ have the continuous second derivative in $t \in \Xi$.

A5 The kernel $K(\cdot)$ is a symmetric probability density function, and is a bounded variation function on its support.

A6 The bandwidth $h$ satisfies $n h^{2} / \log ^{2} n \rightarrow \infty$ and $n h^{8} \rightarrow 0$.

With the assumptions above, we are ready to give the main results. The following theorem gives the asymptotic distribution of $\mathscr{L} \mathscr{R}(\beta)$.

Theorem 3.1. Assume that the A1-A6 hold, if $\beta$ is the true value of the parameter, then

$$
\mathscr{L} \mathscr{R}(\beta) \stackrel{\mathscr{D}}{\rightarrow} \chi_{p}^{2} \quad \text { as } n \rightarrow \infty
$$

where $\stackrel{\mathscr{D}}{\rightarrow}$ denotes the convergence in distribution and $\chi_{p}^{2}$ is a chi-square distribution with $p$ degrees of freedom.

As a consequence of the theorem, the confidence regions for the parameter component $\beta$ can be constructed by (23). More precisely, for any $0<\alpha<1$, let $C_{\alpha}$ be such that $p\left(\chi_{p}^{2}>C_{\alpha}\right) \leq 1-\alpha$. Then

$$
\mathscr{H}(\alpha)=\left\{\beta \in R^{p}: \mathscr{L} \mathscr{R}(\beta) \leq C_{\alpha}\right\}
$$

constitute a confidence region for $\beta$ with asymptotic coverage $1-\alpha$.

Compared with other methods, the empirical likelihood confidence region does not need to estimate a complicated asymptotic covariance matrix. In addition, our empirical likelihood confidence region is not predetermined to be symmetric so that it can better correspond with the true shape of the underlying distribution.

\section{Simulation Results}

In this section, we shall conduct some simulations to the empirical likelihood (EL) method. The data are generated from

$$
\left\{\begin{aligned}
y_{i j} & =x_{i j} \beta+\theta\left(t_{i j}\right)+\epsilon_{i j} \\
w_{i j} & =x_{i j}+u_{i j}
\end{aligned}\right.
$$

where $w_{i j} \sim N(1,1), \beta=1.5, t_{i j} \sim U(-1,1), \theta(t)=\cos (2 \pi t), \epsilon_{i j} \sim N(0,1)$, $u_{i j}=b e_{i, j-1}+e_{i, j}$ and $e_{i j} \sim N(0,1)$.

In the simulation studies, for each combination of $n_{i}$, and $b$, we draw 2,000 random samples of sizes 50 or 100 from the above model, respectively. For each sample, a $95 \%$ confidence interval for $\beta=1.5$ are computed using our block empirical likelihood method. The kernel function is taken as the Gauss kernel 
$K_{h}(t)=\frac{1}{\sqrt{2 \pi} h} \exp \left(-(t)^{2} / 2 h^{2}\right)$. The "leave-one-sample-out" method is used to select the bandwidth $h$. We define the score of $h$ as follows

$$
C V(h)=\frac{1}{n} \sum_{i=1}^{n} \sum_{j=1}^{n_{i}}\left\{Y_{i j}-X_{i j}^{\tau} \check{\beta}_{-i}-\check{\theta}_{-i}\left(t_{i j}\right)\right\}^{2}-\check{\beta}_{-i}^{\tau} \Sigma_{v v} \check{\beta}_{-i}
$$

Then cross-validation smoothing parameter $h_{C V}$ is the minimizer of $C V(h)$. That is,$h_{C V}=\arg \min _{h} C V(h)$. Some representative coverage probabilities are reported in Table 1.

\begin{tabular}{|c|c|c|c|c|}
\hline $\mathrm{k}$ & \multicolumn{2}{|c|}{ Number of replicates } & $\mathrm{CP}(\%)$ & $\mathrm{AL}$ \\
\hline & & $b=0.2$ & & \\
\hline 50 & $n_{1}=\cdots=n_{25}=3$ & $n_{26}=\cdots=n_{50}=3$ & 94.68 & 0.3204 \\
\hline 50 & $n_{1}=\cdots=n_{25}=3$ & $n_{26}=\cdots=n_{50}=2$ & 93.83 & 0.3426 \\
\hline 100 & $n_{1}=\cdots=n_{50}=3$ & $n_{51}=\cdots=n_{100}=3$ & 95.37 & 0.2742 \\
\hline 100 & $n_{1}=\cdots=n_{50}=3$ & $\begin{array}{r}n_{51}=\cdots=n_{100}=2 \\
b=0.4\end{array}$ & 95.16 & 0.2869 \\
\hline 50 & $n_{1}=\cdots=n_{25}=3$ & $n_{26}=\cdots=n_{50}=3$ & 94.34 & 0.3218 \\
\hline 50 & $n_{1}=\cdots=n_{25}=3$ & $n_{26}=\cdots=n_{50}=2$ & 93.97 & 0.3419 \\
\hline 100 & $n_{1}=\cdots=n_{50}=3$ & $n_{51}=\cdots=n_{100}=3$ & 95.12 & 0.2983 \\
\hline 100 & $n_{1}=\cdots=n_{50}=3$ & $n_{51}=\cdots=n_{100}=2$ & 95.03 & 0.2995 \\
\hline
\end{tabular}

Table 1: Coverage probabilities(CP) and average lengths(AL) of the confidence intervals for $\beta=1.5$ and $\sigma^{2}=0.2$

\section{Proof of the Main Results}

In order to prove the main results, we first introduce several lemmas. The following notations will be used in the proof of the lemmas and theorems. Let $u_{k}=\int t^{k} K(t) d t, v_{k}=\int t^{k} K^{2}(t) d t, k=0,1,2,4, c_{n}=h^{2}+\left(\frac{\log n}{n h}\right)^{\frac{1}{2}}$.

Lemma 5.1. Let $\left(X_{1}, Y_{1}\right), \ldots,\left(X_{n}, Y_{n}\right)$ be i.i.d random vector, where $Y_{i}$ is scalar random variable. Further, assume that $E\left|Y_{1}\right|^{s}<\infty, \sup _{x} \int|y|^{s} f(x, y) d y<$ $\infty$, where $f(\cdot, \cdot)$ denotes the joint density of $(X, Y)$. Let $K(\cdot)$ be a bounded positive function with a bounded support, satisfying a Lipschitz condition. Given that $n^{2 \epsilon-1} h \rightarrow \infty$ for some $\epsilon<1-s^{-1}$, then

$$
\sup _{x}\left|\frac{1}{n} \sum_{i=1}^{n}\left\{K_{h}\left(X_{i}-x\right) Y_{i}-E\left[K_{h}\left(X_{i}-x\right) Y_{i}\right]\right\}\right|=O_{p}\left(\left\{\frac{\log \left(\frac{1}{h}\right)}{n h}\right\}^{\frac{1}{2}}\right)
$$


Lemma 5.2. Let $\epsilon_{i}, i=1, \ldots, n$, be a sequence of multi-independent random variate with $E\left(\epsilon_{i}\right)=0$ and $E\left(\epsilon_{i}^{2}\right)<c<\infty$. Then

$$
\max _{1 \leq k \leq n}\left|\sum_{i=1}^{k} \epsilon_{i}\right|=O_{p}(\sqrt{n} \log n)
$$

Further, let $\left(j_{1}, \ldots, j_{n}\right)$ be a permutation of $(1, \ldots, n)$. Then we have

$$
\max _{1 \leq k \leq n}\left|\sum_{i=1}^{k} \epsilon_{j_{i}}\right|=O_{p}(\sqrt{n} \log n) .
$$

Lemma 5.3. Let $D_{1}, \ldots, D_{n}$ be i.i.d random variables. If $E\left|D_{i}\right|^{s}$ are uniformly bounded for $s>1$, then we have

$$
\max _{1 \leq i \leq n}\left|D_{i}\right|=o\left(n^{\frac{1}{s}}\right)
$$

Lemma 5.4. Suppose that the A1-A6 hold, we have

$$
\begin{gathered}
\frac{1}{\sqrt{n}} \sum_{i=1}^{n} \check{\eta}_{i}(\beta) \stackrel{\mathscr{D}}{\rightarrow} N(0, \Sigma) \\
\frac{1}{n} \sum_{i=1}^{n} \check{\eta}_{i}(\beta) \check{\eta}_{i}^{\tau}(\beta) \stackrel{\mathscr{P}}{\longrightarrow} \Sigma \\
\max _{1 \leq x \leq n}\left\|\check{\eta}_{i}(\beta)\right\|=O_{p}\left(n^{1 / 2}\right) .
\end{gathered}
$$

Proof of Theorem 3.1. From (29)-(31), using the same arguments as were used in the proof of Owen [10], we have

$$
\|\lambda\|=O_{p}\left(n^{-1 / 2}\right)
$$

where $\lambda$ is defined in (18). Then, we have

$$
0=\frac{1}{n} \sum_{i=1}^{n} \frac{\check{\eta}(\beta)}{1+\lambda^{\tau} \check{\eta}_{i}(\beta)}=\frac{1}{n} \sum_{i=1}^{n} \check{\eta}_{i}(\beta)-\frac{1}{n} \sum_{i=1}^{n} \check{\eta}_{i}(\beta) \check{\eta}_{i}^{\tau}(\beta) \lambda+\frac{1}{n} \sum_{i=1}^{n} \frac{\check{\eta}_{i}(\beta)\left(\lambda^{\tau} \check{\eta}_{i}(\beta)\right)^{2}}{1+\lambda^{\tau} \check{\eta}_{i}(\beta)}
$$

By using Lemmas 5.4, we obtain

$$
\sum_{i=1}^{n}\left(\lambda^{\tau} \check{\eta}_{i}(\beta)\right)^{2}=\sum_{i=1}^{n} \lambda^{\tau} \check{\eta}_{i}(\beta)+O_{p}(1)
$$




$$
\lambda=\left[\sum_{i=1}^{n} \check{\eta}_{i}(\beta) \check{\eta}_{i}^{\tau}(\beta)\right]^{-1} \sum_{i=1}^{n} \check{\eta}_{i}(\beta)+o_{p}\left(n^{-1 / 2}\right)
$$

Applying the Taylor expansion to (19), we get that

$$
\mathscr{L} \mathscr{R}(\beta)=2 \sum_{i=1}^{n}\left[\lambda^{\tau} \check{\eta}_{i}(\beta)-\frac{1}{2}\left(\lambda^{\tau} \check{\eta}_{i}(\beta)\right)^{2}\right]+o_{p}(1)
$$

Hence, together with (32), we have

$$
\mathscr{L} \mathscr{R}(\beta)=\left[\frac{1}{\sqrt{n}} \sum_{i=1}^{n} \check{\eta}_{i}(\beta)\right]^{\tau}\left[\frac{1}{\sqrt{n}} \sum_{i=1}^{n} \check{\eta}_{i}(\beta) \check{\eta}_{i}^{\tau}(\beta)\right]^{-1}\left[\frac{1}{\sqrt{n}} \sum_{i=1}^{n} \check{\eta}_{i}(\beta)\right]+o_{p}(1)
$$

Together with Lemmas 5.4, this proves Theorem 3.1.

\section{Acknowledgments}

The authors would like to thank the anonymous referees for their useful and helpful suggestion that have improve this paper.

\section{References}

[1] T. J. Engle, C. W. J. Granger, J. Rice, A. Weiss, Semiparametric estimates of the relation between weather and electricity sales, J. Am. Stat. Assoc., 81, No. 394 (1986), 310-320, doi: 10.1080/01621459.1986.10478274.

[2] Z. Hu, N. Wang, R. J. Carroll, Profile-kernel versus backfitting in the partially linear models for longitudinal/clustered data, Biometrika, 91, No. 2 (2004), 251-262, doi: 10.1093/biomet/91.2.251.

[3] N. Heckman, Spline smoothing in partly linear models, J. Roy. Statist. Soc. Ser. B, 48, No. 6 (1986), 244-248.

[4] H. Chen, H. J. Shiau, A two-stage spline smoothing method for partially linear model, J. Stat. Plann. Inference, 27, No. 2 (1991), 187-201, doi: 10.1016/0378-3758(91)90015-7.

[5] H. Liang, W. Härdle, R. J. Carroll,Estimation in a semiparametric partially linear errors-in-variables model, Ann. Stat., 27, No. 5 (1999), 1519-1535, doi: 10.1214/aos/1017939140. 
[6] H. Liang, Asymptotic normality of parametric part in partially linear models with measurement error in the nonparametric part, J. Stat. Plann. Inference, 86, No. 1 (2000), 51-62, doi: 10.1016/S0378-3758(99)00093-2.

[7] L. Zhu, H. Cui, Semiparametric regression model with errors in variables, Scan. J. Statist., 30, No. 2 (2003), 429-442, doi: 10.1111/1467-9469.00340.

[8] A. B. Owen, Empirical likelihood ratio confidence intervals for a single functional, Biometrika, 75, No. 2 (1988), 237-249, doi: 10.1093/biomet/75.2.237.

[9] A. B. Owen, Empirical likelihood ratio confidence regions, Ann. Stat., 18, No. 1 (1990), 90-120, doi: 10.1214/aos/1176347494.

[10] A. B. Owen, Empirical likelihood for linear models, Ann. Stat., 19, No. 4 (1991), 1725-1747, doi: 10.1214/aos/1176348368.

[11] J. Shi, T. Lau, Empirical likelihood for partially linear models, J. Multivariate Anal., 72, No. 1 (2000), 132-148, doi: http:10.1006/jmva.1999.1866.

[12] Q. Wang, B. Jing, Empirical likelihood for partial linear models, Ann. Inst. Statist. Math., 55, No. 3 (2003), 585-595, doi: 10.1007/BF02517809.

[13] L. Xue, L. Zhu, Empirical likelihood semiparametric regression analysis for longitudinal data, Biometrika, 94, No. 4 (2007), 921-937, doi: 10.1093/biomet/asm066.

[14] G. Li, L. Xue, Empirical likelihood confidence region for the parameter in a partially linear errors-in-variables model, Commun. Stat-Theor. M., 37, No. 10 (2008), 1552-1564, doi: 10.1080/03610920801893913.

[15] H. Cui, S. Chen, Empirical likelihood confidence region for parameter in the errors-in-variables models, J. Multivariate Anal., 84, No. 1 (2003), 101-115, doi: 10.1016/S0047-259X(02)00017-9.

[16] J. You, G. Chen, Y, Zhou, Block empirical likelihood for longitudinal partially linear regression models, Can. J. Stat., 34, No. 1 (2006), 79-96, doi: $10.1002 /$ cjs.5550340107.

[17] L. Xue, L. Zhu, Empirical likelihood-based inference in a partially linear model for longitudinal data, Science in China Series A: Mathematics, 51, No. 1 (2008), 115-130, doi: 10.1007/s11425-008-0020-4. 
[18] X. Lu, Empirical likelihood for heteroscedastic partially linear models, J. Multivariate Anal., 100, No. 3 (2009), 387-396, doi: http:10.1016/j.jmva.2008.05.006.

[19] W. A. Fuller, Measurement Error Models, Wiley, New York (1987). 